\title{
Expression of the Inhibitory CD200 Receptor Is Associated with Alternative Macrophage Activation
}

\author{
Nathalie Koning ${ }^{a}, b \quad$ Marco van Eijk ${ }^{c}$ Walter Pouwels ${ }^{b}$ Michael S.M. Brouwer ${ }^{b}$ \\ David Voehringer $^{\mathrm{e}}$ Inge Huitinga ${ }^{\mathrm{a}} \mathrm{d}$ Robert M. Hoek ${ }^{\mathrm{b}}$ Geert Raes $^{\mathrm{f}}$ \\ Jörg Hamann ${ }^{b}$ \\ a Department of Neuroimmunology, Netherlands Institute for Neuroscience, Institute of the Royal Netherlands \\ Academy for Arts and Sciences, and Departments of ${ }^{b}$ Experimental Immunology and ${ }^{\mathrm{C}}$ Medical Biochemistry, \\ Academic Medical Center, and d Netherlands Brain Bank, Amsterdam, The Netherlands; ${ }^{\circ}$ Department of Medicine, \\ Institute for Immunology, University of Munich, Munich, Germany; ${ }^{f}$ Laboratory of Cellular and Molecular Immunology, \\ Vrije Universeit Brussel, and VIB, Department of Molecular and Cellular Interactions, Brussels, Belgium
}

\section{Key Words}

IL-4 - Immune inhibition - Macrophage polarization •

Parasite infection - Type 2 responses

\begin{abstract}
Classical macrophage activation is inhibited by the CD200 receptor (CD200R). Here, we show that CD200R expression was specifically induced on human in vitro polarized macrophages of the alternatively activated M2a subtype, generated by incubation with IL-4 or IL-13. In mice, peritoneal M2 macrophages, elicited during infection with the parasites Taenia crassiceps or Trypanosoma brucei brucei, expressed increased CD200R levels compared to those derived from uninfected mice. However, in vitro stimulation of mouse peritoneal macrophages and T. crassiceps infection in IL-4-/- and IL-4R-/- mice showed that, in contrast to humans, induction of CD200R in mice was not IL-4 or IL-13 dependent. Our data identify CD200R as a suitable marker for alternatively activated macrophages in humans and corroborate observations of distinct species- and/or site-specific mechanisms regulating macrophage polarization in mouse and man.
\end{abstract}

Copyright $\odot 2009$ S. Karger AG, Basel
(C) 2009 S. Karger AG, Basel

$1662-811 \mathrm{X} / 10 / 0022-0195 \$ 26.00 / 0$

Fax +41613061234

E-Mail karger@karger.ch

www.karger.com
Accessible online at:

www.karger.com/jin
Macrophages are versatile players in the immune system. They effectively neutralize pathogens and harmful endogenous products via phagocytosis but also contribute to efficient immunity by production and secretion of multiple cytokines and chemokines. Next to effector functions, macrophages possess important activities during the resolution phase of an immune response and at sites where antigenic material needs to be ignored (lung and colon) or tolerated (eye, brain, ovary, and testis). The different macrophage activities result from the initiation of distinct gene expression programs [1-5]. IFN $\gamma$ and TNF induce classical activation of macrophages. These macrophages, also known as M1 cells, produce high amounts of pro-inflammatory cytokines, nitric oxide, and reactive oxygen species and efficiently clear pathogens either directly or by facilitating $\mathrm{T}_{\mathrm{H}} 1$ immune responses. In contrast, stimulation with IL-4 or IL-13 triggers pathways that program macrophages to exert antiinflammatory functions. These alternatively activated macrophages, also known as M2a cells, are considered to be involved in $\mathrm{T}_{\mathrm{H}} 2$ immune responses and wound healing. Finally, IL-10 and glucocorticoids give rise to regulatory macrophages that can inhibit immune responses, 
the so-called M2c cells. Although macrophage activation generates a wide spectrum of subpopulations with overlapping activities, it is useful to identify functionally divergent cells since growing evidence demonstrates that these are involved in various pathologies $[4,5]$. Most of the current knowledge about surface markers on activated macrophages is derived from murine models, but data from human studies are scarce. Based on functional aspects, we reasoned that immune-inhibitory receptors such as the membrane glycoprotein CD200 receptor (CD200R) might be associated with M2 cells. CD200R, mainly expressed on myeloid cells and on a subset of $\mathrm{T}$ and $B$ cells [6-8], inhibits inflammatory macrophage activation as illustrated by studies showing that absence of its ligand CD200 aggravates, accelerates, or increases the susceptibility of inflammatory models, such as experimental autoimmune encephalomyelitis, uveoretinitis, and collagen-induced arthritis [7, 9]. Indeed, CD200R signaling profoundly inhibits the MAP kinases p38, ERK, and JNK, the common signaling pathways involved in classical activation of macrophages [10]. As M2 cells can mediate a $\mathrm{T}_{\mathrm{H}} 2$ immune response by producing and secreting anti-inflammatory mediators and reducing inflammatory cytokines like TNF, it is interesting that some of these effects have also been reported for CD200R [11]. However, the expression pattern of CD200R or other immune-inhibitory molecules on polarized macrophages has never been studied thoroughly.

To determine the expression of CD200R on polarized macrophages, we subjected human monocytes directly after purification from peripheral blood to a panel of proand anti-inflammatory stimuli for 3 days, as described previously [12], to initiate either M1 activation (IFN $\gamma$, IL$1 \beta$, and TNF) or M2 activation (IL-4, IL-13, and IL-10; all PeproTech, London, UK, or dexamethasone; Sigma Aldrich, Zwijndrecht, The Netherlands; fig. 1). Additional pro- and anti-inflammatory cells, corresponding to M1/ M2 macrophages, were created by culturing monocytes in the presence of GM-CSF and M-CSF, respectively (both PeproTech) [13]. Analysis of CD200R mRNA expression in these cells, performed by quantitative realtime PCR (qPCR) [14], revealed a significant induction after stimulation with IL-4 or IL-13 (fig. 1). At protein level, determined by flow cytometry using Alexa 674labeled anti-human CD200R (Serotec, Oxford, UK), CD200R expression was robustly increased by IL-4 stimulation, modestly increased by IL-13, and slightly but not significantly following IL-10 and the glucocorticoid dexamethasone (fig. 1). These results indicate that CD200R is specifically upregulated on alternatively activated M2a macrophages, which are at present most commonly identified by expression of the mannose receptor (MR, CD206) [1]. We therefore compared the expression pattern of CD200R with that of MR mRNA by qPCR and protein by flow cytometry, using APC-labeled anti-MR (BD Biosciences, San Jose, Calif., USA), in the same series of experiments. MR was indeed induced by IL- 4 and IL-13, but also by the M1 activator GM-CSF, confirming previously reported data [15], and to some extent by IL-1 $\beta$, although the latter was not significant (fig. 1). Different from M2a cells are M2c cells that are induced by IL-10 and glucocorticoids, and are characterized by expression of the scavenger receptor CD163 [3]. As expected, CD163 mRNA and protein (PE-labeled anti-CD163, BD Biosciences) was induced only by IL-10 and dexamethasone and not by IL4 or IL-13 (fig. 1), confirming the M2c specificity of this marker. To further validate our findings, we cultured freshly isolated monocytes for an extended period of 7 days, the last 3 days being in the presence of either IL-4 or IL-10. Compared to cells that were cultured for 3 days (fig. 1), this procedure induced a further increase in CD200R mRNA expression following IL-4 stimulation (fold induction $14.2 \pm 2.6, \mathrm{n}=8$ ). Similar to CD200R, the increase in MR expression was also much stronger (fold induction $14.4 \pm 2.1, \mathrm{n}=8$ ) while CD163 induction by IL-10 was not further upregulated compared to the 3-day cultured cells. As expected, CD200R and MR expression was not upregulated by IL-10 nor was CD163 induced by

Fig. 1. Expression of CD200R by human macrophages maturated for 3 days under polarizing conditions. Human primary monocytes were isolated from PBMCs and differentiated into M1 macrophages by stimulation with IFN $\gamma(50 \mathrm{ng} / \mathrm{ml}), \mathrm{IL}-1 \beta(40 \mathrm{ng} / \mathrm{ml})$, TNF (50 ng/ml), or GM-CSF (50 ng/ml) or into M2 macrophages by stimulation with M-CSF ( $25 \mathrm{ng} / \mathrm{ml}), \mathrm{IL}-4$ (40 ng/ml), IL-13 (50 $\mathrm{ng} / \mathrm{ml})$, IL-10 (50 ng/ml), or dexamethasone (Dex; $2 \mathrm{nM})$. After $72 \mathrm{~h}, \mathrm{mRNA}$ and protein expression of CD200R, MR, CD163, and TREM2 were determined by qPCR and flow cytometry, respectively. Transcript data (a) are depicted as mean fold induction \pm SEM compared to macrophages cultured in medium only [number of donors $(\mathrm{n})=3 ; 2$ independent experiments]. Protein data (b) are depicted as mean fluorescence intensity $\left(\mathrm{GEO}_{\text {mean }}\right) \pm$ SEM (CD200R, $\mathrm{n}=11 ; \mathrm{MR}, \mathrm{n}=5$; CD163, $\mathrm{n}=6$; TREM2, $\mathrm{n}=3$; 2-6 independent experiments). Representative histograms (c) show protein expression levels of cells cultured with IL-4 (bold line), IL-10 (thin line), or medium alone (grey), with geometric means indicated by the corresponding numbers (isotype controls left and antibodies right). ${ }^{*} 0.01<\mathrm{p} \leq 0.05 ;{ }^{*} 0.001<\mathrm{p} \leq 0.01$; ${ }^{* * *} \mathrm{p}<0.001$ (one-way ANOVA and post-hoc Dunnett's multiple comparison test). 

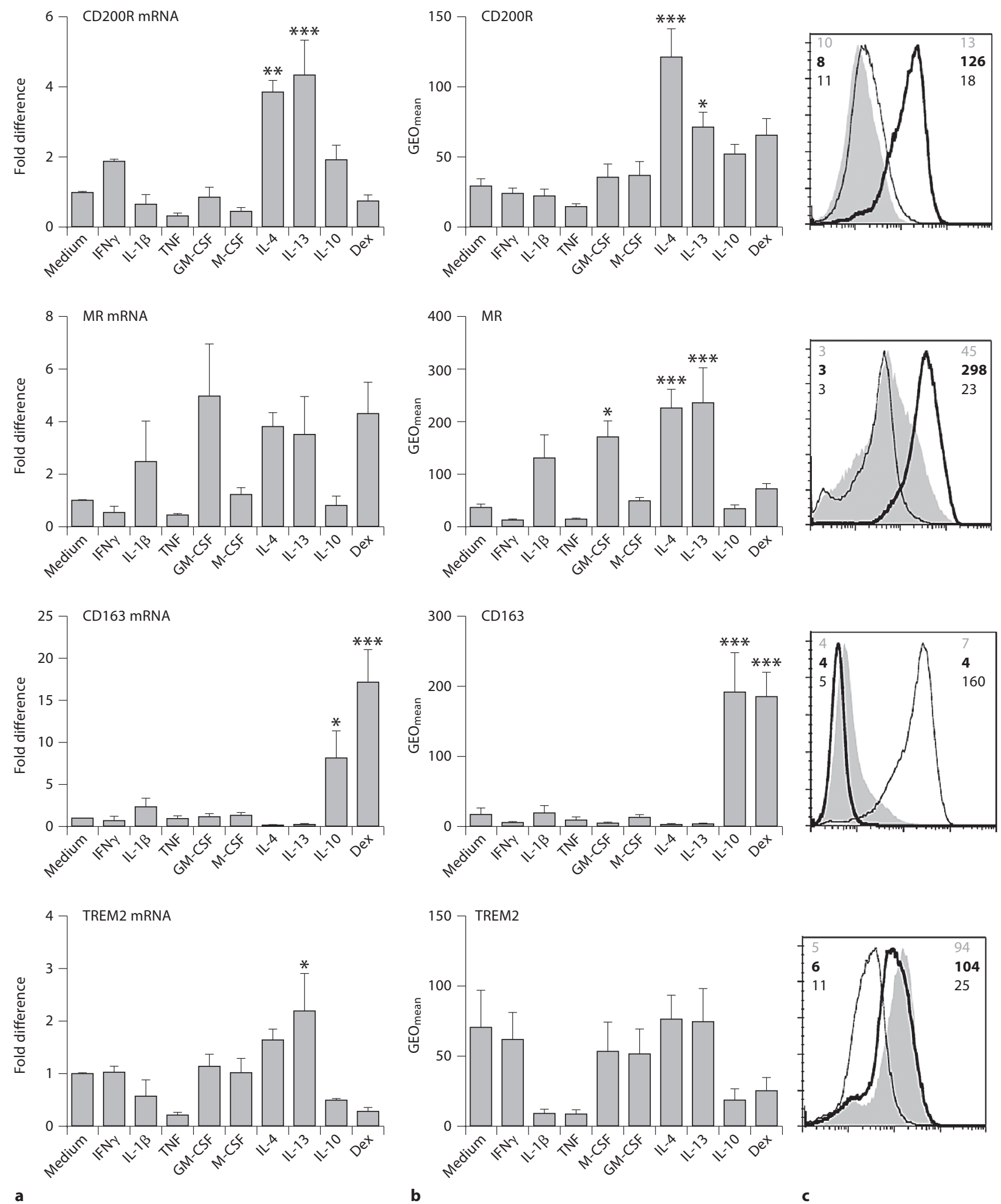
Table 1. Protein expression levels of CD200R on human myeloid cells

\begin{tabular}{ll}
\hline Cell type (n) & $\begin{array}{l}\text { Fold difference of geometric } \\
\text { MFI over control Ig } \\
\text { (mean } \pm \text { SEM) }\end{array}$ \\
\hline Macrophages & \\
$\quad$ Monocyte-derived macrophages & \\
$\quad$ Polarized with IL-4 (11) & $49.6 \pm 9.6$ \\
$\quad$ Non-polarized (11) & $11.6 \pm 2.5$ \\
Splenic macrophages (3) & $10.1 \pm 1.4$ \\
Monocytes & $20.2 \pm 2.2$ \\
$\quad$ CD16- monocytes (5) & $10.6 \pm 0.8$ \\
CD16+ monocytes (5) & $12.4 \pm 1.3$ \\
Dendritic cells & $1.3 \pm 0.1$ \\
BDCA1+ myeloid DC (4) & \\
BDCA4+ plasmacytoid DC (4) & $90.9 \pm 9.1$ \\
Granulocytes & $24.5 \pm 5.2$ \\
Basophils (4) & $12.5 \pm 1.1$ \\
Neutrophils (5) & \\
Eosinophils (5) &
\end{tabular}

MFI = Mean fluorescence intensity; $\mathrm{n}=$ number of donors.

IL-4 (data not shown). Thus, whereas CD163 is an excellent marker for M2c cells, CD200R is abundantly expressed on human M2a cells, with even higher specificity than MR, and appears to be a novel reliable marker for this type of macrophage in vitro.

We next tested whether two other inhibitory molecules expressed on myeloid cells, TREM2 and SIRP $\alpha$ [16$18]$, are also primarily expressed by alternatively activated macrophages. A significant induction of TREM2 was found after IL-13 stimulation at mRNA level, but not at protein level (biotinylated anti-TREM2, PeproTech; fig. 1). IL-4 did not induce TREM2 expression. Expression of SIRP $\alpha$, determined at the mRNA level, was not induced by any of the investigated stimuli (data not shown), indicating that induction of immune-inhibitory molecules is not a common phenotype of experimentally polarized human M2 macrophages, and seems restricted to CD200R.

Because CD200R is present on various leukocytes [19], we compared its expression levels on in vitro polarized macrophages with that on primary myeloid cells. Table 1 shows that CD200R is most abundant on basophilic granulocytes, which is in agreement with previous studies [20]. CD200R expression on monocytes, myeloid dendritic cells, and splenic macrophages was lower compared to M2a cells generated in vitro.
Experimental infection of mice with parasites is known to provoke strong type 2 immune responses correlating with an M2 signature of the elicited macrophages [21]. To test whether CD200R is expressed on M2 cells in vivo, we analyzed peritoneal macrophages isolated from animals infected with either the helminth Taenia crassiceps or the protozoan Trypanosoma brucei brucei, inoculated as described previously [21]. Indeed, macrophages from $T$. crassiceps-infected mice (C57BL/6 and $\mathrm{BALB} / \mathrm{c}$ ) showed increased CD200R mRNA expression compared to those derived from uninfected mice (fig. 2a). Mice infected with phospholipase C-deficient (PLC-/-) T. b. brucei display a switch from a type 1 cytokine milieu during the early stage of the infection to a type 2 environment in the chronic phase of the disease [21]. CD200R expression was unaltered or even slightly decreased on peritoneal macrophages derived from the acute phase, but robustly upregulated on macrophages derived from the chronic phase (fig. 2a). In both parasite models, regulation of CD200R expression correlated well with the established mouse M2 markers arginase-1 (Arg-1) and 'found in inflammatory zone 1' (FIZZ1) [21] (online suppl. table S1, www.karger.com/doi/10.1159/000252803). These results indicate that CD200R is also induced on mouse M2 macrophages in vivo.

We next determined whether CD200R expression on mouse M2 macrophages was induced by IL-4 or IL-13, as shown in our previous studies in human macrophages. Surprisingly, IL-4 (R \& D Systems, Minneapolis, Minn., USA) and IL-13 (Sanquin, Amsterdam, The Netherlands) failed to induce CD200R mRNA expression in resident peritoneal macrophages from C57BL/6 mice after 24 (data not shown) and $48 \mathrm{~h}$ in vitro (fig. $2 \mathrm{~b}$ ), whereas induction of alternatively activated macrophages was confirmed by upregulation of Arg-1 and FIZZ1 (online suppl. table S1). IL-21 was recently reported to augment alternative macrophage activation [22]. In line with these data, we found that IL-21 (Sanquin) alone did not induce Arg1 or FIZZ1, but enhanced the expression of these markers when added to IL-4 plus IL-13 (online suppl. table S1). After stimulation with this potent combination of cytokines, again, no upregulation of CD200R was detected (fig. 2b). Stimulation with IL-10 (PeproTech) had no effect on CD200R expression (fig. 2b) and IFNy (PeproTech) seemed to decrease CD200R expression levels. Also, in thioglycolate-elicited peritoneal macrophages from either $\mathrm{C} 57 \mathrm{BL} / 6$ ( $\mathrm{T}_{\mathrm{H}} 1$-prone) or BALB/c $\left(\mathrm{T}_{\mathrm{H}} 2\right.$-prone) mice, IL-4 or IL-10 did not affect CD200R expression (online suppl. table S1). In vivo, we confirmed that the elevated CD200R expression in M2 cells was indeed IL-4 


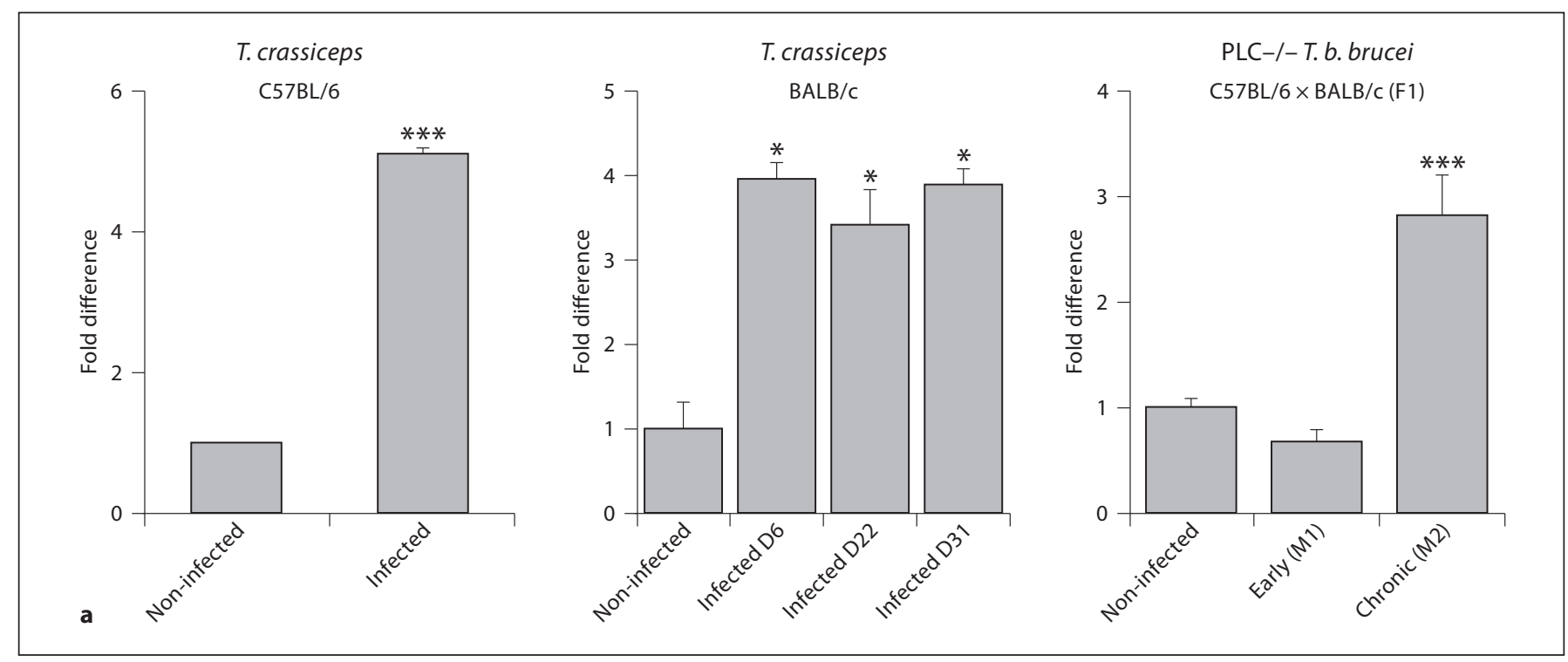

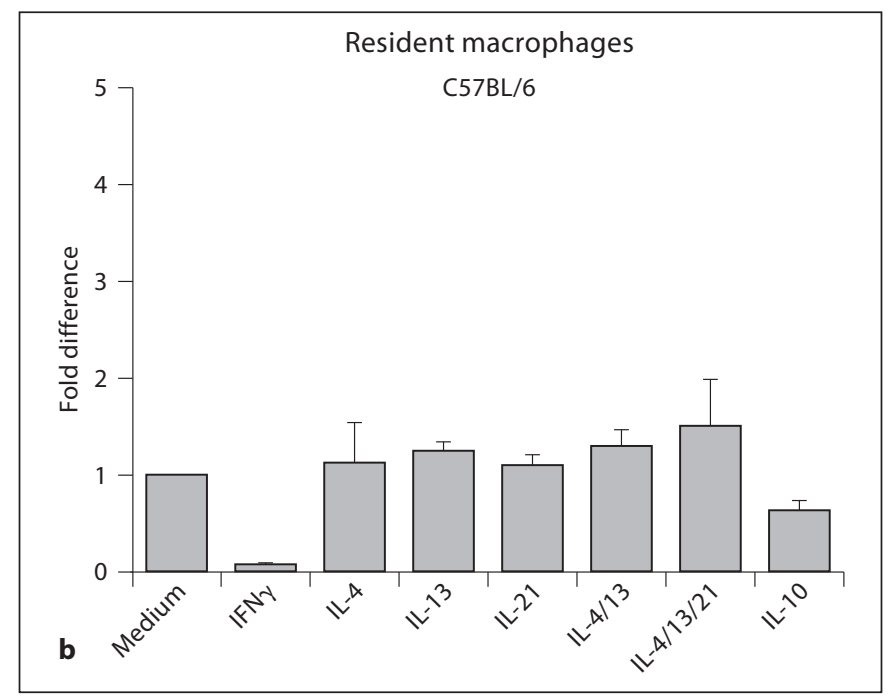

Fig. 2. CD200R expression in mouse alternatively activated macrophages in vivo and in vitro. a Transcription of CD200R was determined by qPCR in peritoneal macrophages isolated from C57BL/6 mice 4 weeks after infection with T. crassiceps (left panel) and from BALB/c mice 6, 22, and 31 days after infection (middle panel). CD200R expression was also determined in peritoneal macrophages isolated from $\mathrm{C} 57 \mathrm{BL} / 6 \times \mathrm{BALB} / \mathrm{c}(\mathrm{F} 1)$ mice in the early (2 weeks) and chronic (3-4 months) stage of infection with PLC-I- T. b. brucei (right panel; $\mathrm{n}=2-3$ mice per group). $\mathbf{b}$ Induction in CD200R mRNA in resident peritoneal macrophages iso-

independent as no abrogation of CD200R induction was observed in IL-4-/- or IL-4R-/- mice infected with $T$. crassiceps (fig. 2c). The exact conditions causing CD200R induction in the mouse are currently unknown, but may involve site-specific combinations of cytokines including

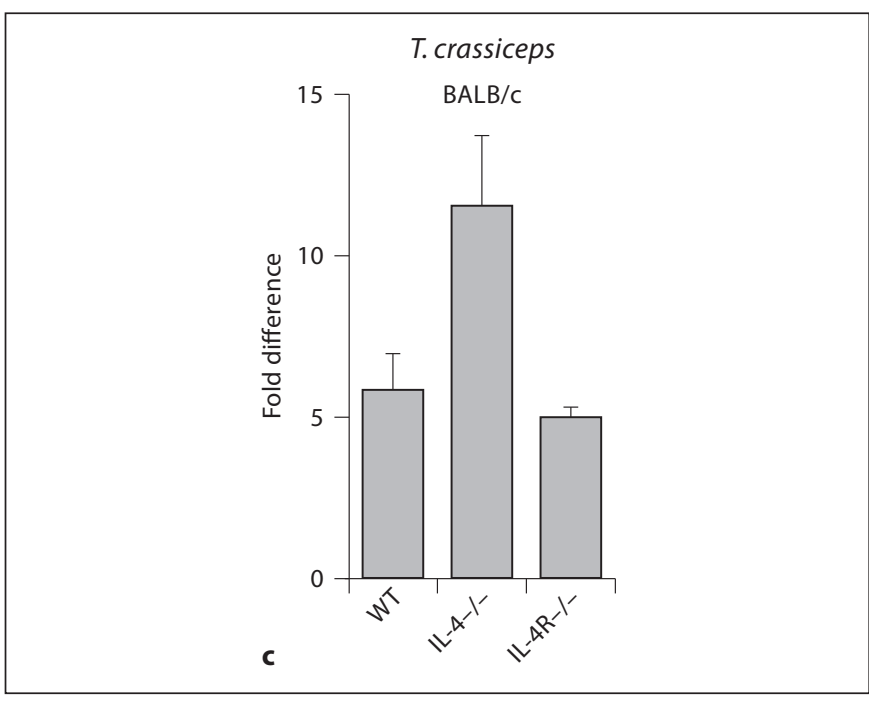

lated from C57BL/6 mice stimulated with IFN $\gamma$, IL-4, IL-13, IL-21, IL-10 (all $50 \mathrm{ng} / \mathrm{ml}$ ), or combinations thereof for $48 \mathrm{~h}(\mathrm{n}=3$ experiments, cells are pooled from 4-5 mice per experiment). c Induction of CD200R mRNA on peritoneal macrophages isolated from BALB/c wild-type, IL-4-/-, and IL-4R-/- mice infected with T. crassiceps. Data are depicted as mean fold induction \pm SEM compared to macrophages from non-infected mice (a), untreated cultures (b), and non-infected wild-type (WT) mice (c; $\mathrm{n}=3$ ). ${ }^{*} 0.01<\mathrm{p} \leq 0.05 ;{ }^{* *} \mathrm{p}<0.001$ (one-way ANOVA and post hoc Dunnett's multiple comparison test).

also inflammatory signals [23]. Interestingly, M2a markers expressed in the dermal environment in the mouse also appear to be independent of IL-4 [24].

In summary, we here demonstrate that the immuneinhibitory molecule CD200R is induced on human mac- 
rophages polarized in vitro following IL-4 or IL-13 stimulation and can be used as a novel cell surface marker for M2a cells in human. Furthermore, CD200R is also expressed on mouse M2 cells, but in contrast to humans, is not induced by classical type 2 cytokines in vitro. CD200R ligation induces immune suppression and will thus contribute to the immune-regulatory capacity of M2 macrophages. By repressing pathways associated with classical macrophage activation [10], CD200R may also be associated with maintaining M2 cells in their polarized state, which enables them to create a milieu that favors $\mathrm{T}_{\mathrm{H}} 2 \mathrm{im}$ - mune responses, and tissue homeostasis and repair. Lack of this regulatory pathway, for instance in CD200-/mice, could then lead to exacerbation of (auto)immune diseases as reported previously $[4,7,9,23]$.

\section{Acknowledgments}

We thank Saskia Scheij for technical assistance. This study was supported by a grant from the Dutch Multiple Sclerosis Research Foundation (MS02-496).

\section{References}

1 Stein M, Keshav S, Harris N, Gordon S: Interleukin 4 potently enhances murine macrophage mannose receptor activity: a marker of alternative immunologic macrophage activation. J Exp Med 1992;176:287-292.

$\checkmark 2$ Goerdt S, Orfanos CE: Other functions, other genes: alternative activation of antigenpresenting cells. Immunity 1999;10:137142.

$\checkmark 3$ Mantovani A, Sica A, Sozzani S, Allavena P, Vecchi A, Locati M: The chemokine system in diverse forms of macrophage activation and polarization. Trends Immunol 2004;25: 677-686.

4 Martinez FO, Helming L, Gordon S: Alternative activation of macrophages: an immunologic functional perspective. Annu Rev Immunol 2009;27:451-483.

5 Mosser DM, Edwards JP: Exploring the full spectrum of macrophage activation. Nat Rev Immunol 2008;8:958-969.

6 Wright GJ, Puklavec MJ, Willis AC, Hoek RM, Sedgwick JD, Brown MH, Barclay AN: Lymphoid/neuronal cell surface OX2 glycoprotein recognizes a novel receptor on macrophages implicated in the control of their function. Immunity 2000;13:233-242.

7 Hoek RM, Ruuls SR, Murphy CA, Wright GJ, Goddard R, Zurawski SM, Blom B, Homola ME, Streit WJ, Brown MH, Barclay AN, Sedgwick JD: Down-regulation of the macrophage lineage through interaction with OX2 (CD200). Science 2000;290:1768-1771.

$>8$ Rijkers ES, de Ruiter T, Baridi A, Veninga H, Hoek RM, Meyaard L: The inhibitory CD200R is differentially expressed on human and mouse $\mathrm{T}$ and $\mathrm{B}$ lymphocytes. Mol Immunol 2008;45:1126-1135.

$\checkmark 9$ Broderick C, Hoek RM, Forrester JV, Liversidge J, Sedgwick JD, Dick AD: Constitutive retinal CD200 expression regulates resident microglia and activation state of inflammatory cells during experimental autoimmune uveoretinitis. Am J Pathol 2002;161:16691677.
10 Zhang S, Cherwinski H, Sedgwick JD, Phillips JH: Molecular mechanisms of CD200 in hibition of mast cell activation. J Immunol 2004;173:6786-6793.

11 Jenmalm MC, Cherwinski H, Bowman EP, Phillips JH, Sedgwick JD: Regulation of myeloid cell function through the CD200 receptor. J Immunol 2006;176:191-199.

12 van Eijk M, van Roomen CP, Renkema GH, Bussink AP, Andrews L, Blommaart EF, Sugar A, Verhoeven AJ, Boot RG, Aerts JM: Characterization of human phagocyte-derived chitotriosidase, a component of innate immunity. Int Immunol 2005; 17:15051512 .

13 Verreck FA, de Boer T, Langenberg DM, Hoeve MA, Kramer M, Vaisberg E, Kastelein R, Kolk A, de Waal-Malefyt R, Ottenhoff TH: Human IL-23-producing type 1 macrophages promote but IL-10-producing type 2 macrophages subvert immunity to (myco)bacteria. Proc Natl Acad Sci USA 2004;101: 4560-4565.

14 Koning N, Bö L, Hoek RM, Huitinga I: Downregulation of macrophage inhibitory molecules in multiple sclerosis lesions. Ann Neurol 2007;62:504-514.

15 Chroneos Z, Shepherd VL: Differential regulation of the mannose and SP-A receptors on macrophages. Am J Physiol 1995;269:L721L726.

16 Turnbull IR, Colonna M: Activating and inhibitory functions of DAP12. Nat Rev Immunol 2007;7:155-161.

17 Kharitonenkov A, Chen Z, Sures I, Wang H, Schilling J, Ullrich A: A family of proteins that inhibit signalling through tyrosine kinase receptors. Nature 1997;386:181-186.
18 Barclay AN, Brown MH: The SIRP family of receptors and immune regulation. Nat Rev Immunol 2006;6:457-464.

19 Wright GJ, Cherwinski H, Foster-Cuevas M, Brooke G, Puklavec MJ, Bigler M, Song Y, Jenmalm M, Gorman D, McClanahan T, Liu MR, Brown MH, Sedgwick JD, Phillips JH, Barclay AN: Characterization of the CD200 receptor family in mice and humans and their interactions with CD200. J Immunol 2003;171:3034-3046.

20 Shiratori I, Yamaguchi M, Suzukawa M, Yamamoto K, Lanier LL, Saito T, Arase H: Down-regulation of basophil function by human CD200 and human herpesvirus- 8 CD200. J Immunol 2005; 175:4441-4449.

21 Ghassabeh GH, De BP, Brys L, Noel W, Van Ginderachter JA, Meerschaut S, Beschin A, Brombacher F, Raes G: Identification of a common gene signature for type II cytokineassociated myeloid cells elicited in vivo in different pathologic conditions. Blood 2006; 108:575-583.

22 Pesce J, Kaviratne M, Ramalingam TR, Thompson RW, Urban JF Jr, Cheever AW, Young DA, Collins M, Grusby MJ, Wynn TA: The IL-21 receptor augments Th2 effector function and alternative macrophage activation. J Clin Invest 2006;116:2044-2055.

23 Snelgrove RJ, Goulding J, Didierlaurent AM, Lyonga D, Vekaria S, Edwards L, Gwyer E, Sedgwick JD, Barclay AN, Hussell T: A critical function for CD200 in lung immune homeostasis and the severity of influenza infection. Nat Immunol 2008;9:1074-1083.

24 Dupasquier M, Stoitzner P, Wan H, Cerqueira D, van Oudenaren A, Voerman JS, DendaNagai K, Irimura T, Raes G, Romani N, Leenen PJ: The dermal microenvironment induces the expression of the alternative activation marker CD301/mMGL in mononuclear phagocytes, independent of IL-4/IL-13 signaling. J Leukoc Biol 2006;80:838-849. 